\title{
Method for the Controlled Burning of Combustible Materials and Analyses of the Combustion Gases
}

\author{
Alan Schriesheim ${ }^{2}$
}

\begin{abstract}
A method is described to burn a given quantity of a combustible material in a fixed amount of air at a selected initial temperature. Analyses of most of the gaseous combustion products were made quickly and comprehensively by means of a mass spectrometer. Hydrogen chloride was not detected with the mass spectrometer because of its adsorption upon the walls of the glass combustion chamber. The concentration of this gas was determined by titrating a water wash of the combustion chamber with a standard silver nitrate solution. Carbon monoxide when present in concentrations below 1,000 parts per million was determined by an indicator method, and when present in concentrations above 1,000 parts per million was determined by the mass spectrometer. Several organic coatings of different chemical composition were burned in a combustion space initially maintained at three temperatures. The lowest temperature $\left(250^{\circ} \mathrm{C}\right)$ produced the smallest variety of combustion gases, whereas the highest temperature $\left(550^{\circ} \mathrm{C}\right)$ produced the largest variety.
\end{abstract}

\section{Introduction}

Organic materials are widely used for various building purposes. When these organic materials are exposed in air to elevated temperatures, gaseous combustion products are formed. The principal combustion products are carbon monoxide, carbon dioxide, and water. Other gases, such as phosgene, hydrogen chloride, ammonia, cyanogen, and sulfur compounds, have been found upon the combustion of chlorinated hydrocarbons, wool, silk, and rubber [1 to 4$].^{3}$

In order to study the gaseous combustion products of these organic materials, several investigators $[1,2,3]$ have burned large built-up specimens, including actual rooms and buildings. Obviously, this procedure is costly and time consuming. Several laboratory methods have been developed $[1,2,5]$, which, however, lacked (a) a quick and comprehensive method for analyzing the gases, and (b) a method for controlling the amount of air per unit of weight of the specimen at any initial temperature.

A laboratory procedure designed to burn or decompose a given quantity of material in a fixed volume of air, at different temperatures, is described. A procedure is also described that utilizes a mass spectrometer to increase the speed and extend the scope of the gas analyses.

\section{Apparatus}

Figure 1 is a schematic diagram of the entire apparatus. Figure 2 is a diagram of the combustion chamber alone.

The combustion chamber, figure 2 , was constructed of Pyrex glass, except for the combustion tube extending through its center. This tube was a 12 -in. length of silica combustion tubing, 1 in. in diameter, that had been cut in half along its axis for approximately half of its length. This combustion tube

\footnotetext{
1 The experimental work was supported by the U. S. Air Force.

3 Figures in brackets indicate the literature references at the end of this paper.
}

was sealed to the walls of the combustion chamber through a graded silica-Pyrex seal.

A strip of mica, 3.5 in. by 1.2 in., was placed on top of the open part of the silica tube, and $20 \mathrm{ft}$ of platinum wire, $0.019 \mathrm{in}$. in diameter and coiled in a $1 / 4$-in.-diameter spiral, was wound around the silica tube and over the mica strip (fig. 2).

A thermocouple was placed below the mica strip, and both thermocouple and platinum lead-in wires entered the combustion chamber through openings that were sealed gas-tight with Wood's metal.

The combustion chamber was attached to a vacuum manifold through the glass spiral and two ball joints (fig. 1). The spiral was constructed of 5 turns of 7-mm Pyrex tubing, each turn having a diameter of 6 in. The combination of spiral and ball joint provided enough movement so that the combustion chamber could be inclined.

\section{Procedures}

Under ordinary conditions, an accidental fire starts at atmospheric pressure. This condition was duplicated in the combustion chamber by allowing the drawoff tube to remain open until the initial temperature was reached, and then closing it.

Because the combustion chamber was allowed to remain open to the atmosphere, different amounts of air were left in the system at each initial temperature. In order to maintain a constant ratio of the weight of the specimen to the amount of air at each initial temperature, the weights of the specimens were adjusted to compensate for the decrease in the amount of air in the system at the higher temperatures.

The ratio of the weight of the specimen to the amount of air in the system at room temperature was chosen as a reference point. The weights of the specimens to be used at the higher temperatures were calculated by means of the relationship

$$
\frac{X_{1}}{X_{2}}=\frac{n_{1}}{n_{2}},
$$




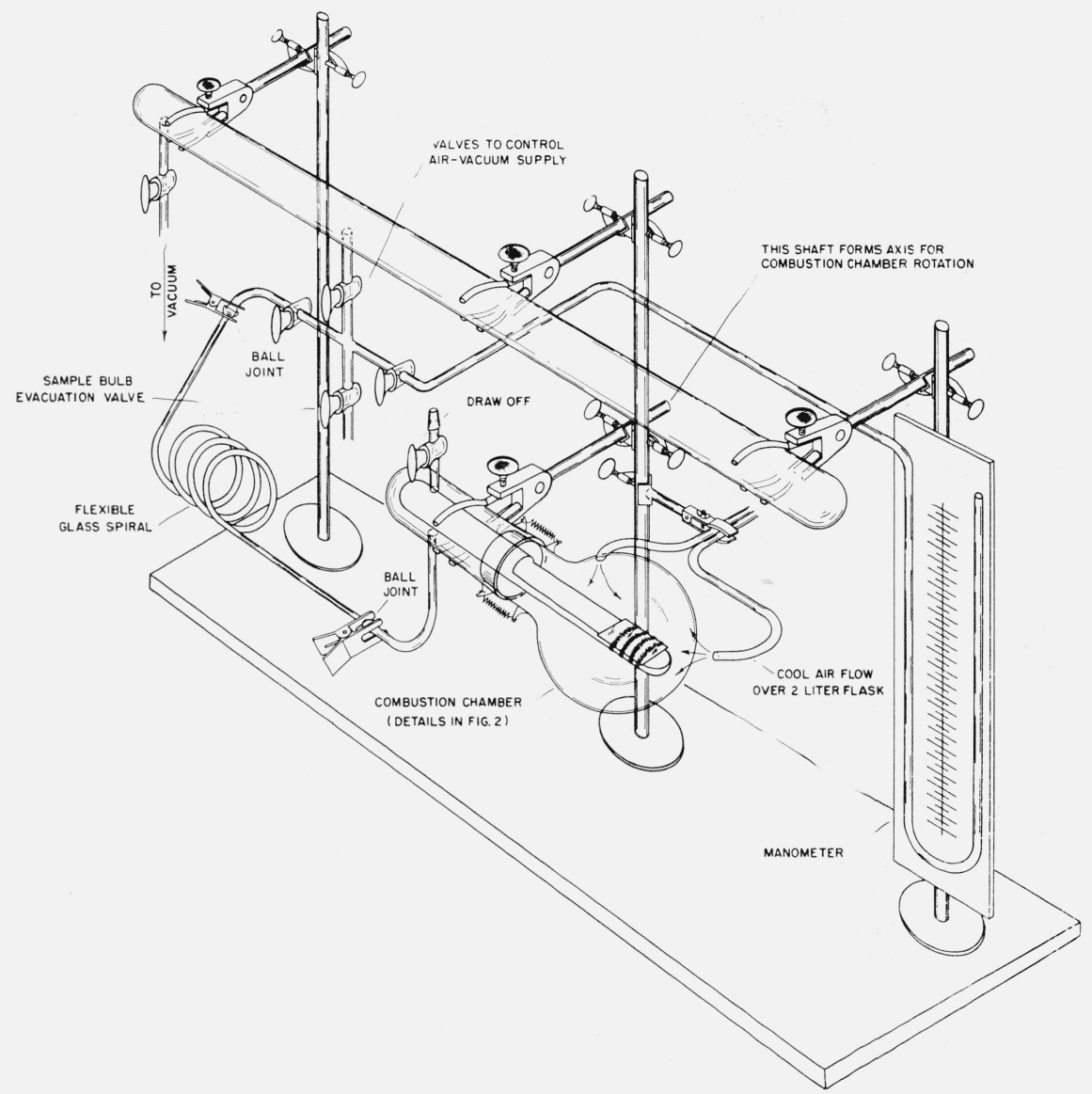

Figure 1. Combustion apparatus.

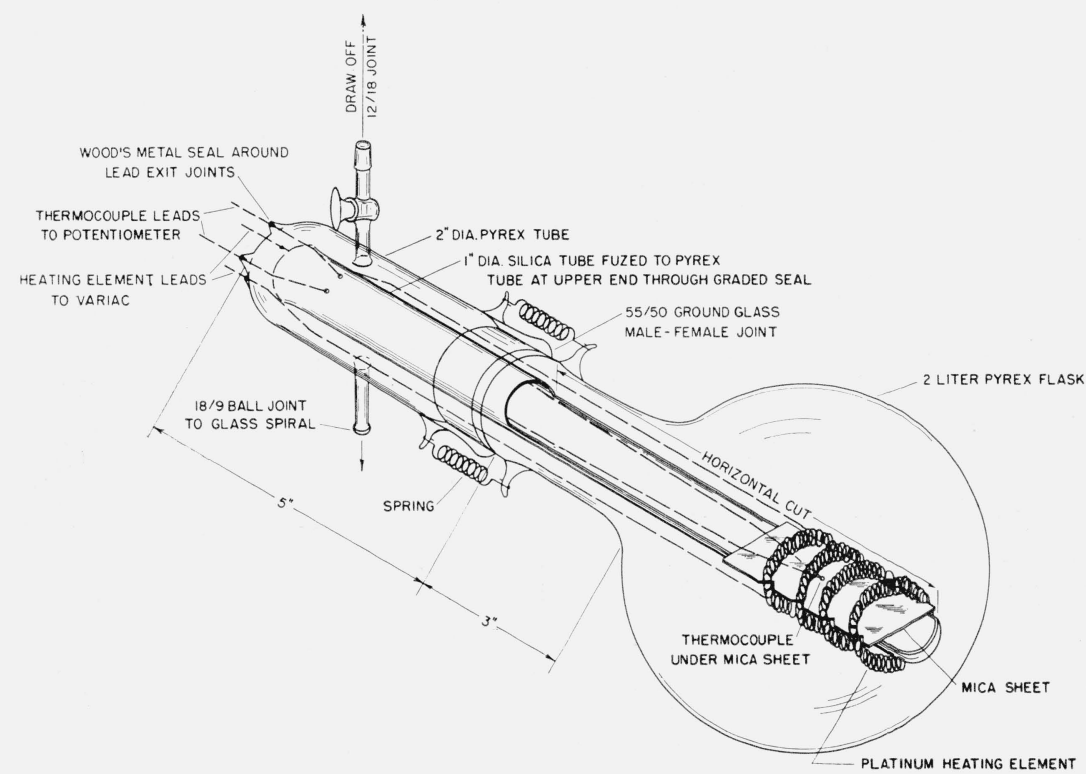

Figure 2. Details of combustion chamber. 
where

$X_{1}=$ the weight of the sample at room temperature.

$X_{2}=$ the weight of the sample at the initial temperature.

$n_{1}=$ the number of moles of air in the system at room temperature.

$n_{2}=$ the number of moles of air in the system at the initial temperature.

The quantity $n_{1}$ can be derived from the perfect-gas equation,

$$
P_{1} V_{1}=n_{1} R T_{1},
$$

because the volume of the system, $V_{1}$, is known $(2,230 \mathrm{ml})$, and $P_{1}$ and $T_{1}$ refer to the atmospheric pressure and room temperature, respectively.

The calculation of $n_{2}$, however, posed some difficulties. Although the initial temperature in the vicinity of the platinum coil was known, the temperature, $T_{2}$, of the entire system, encompassing the heated platinum coil, the air inside the system, and the cooled walls of the combustion chamber, was unknown. Hence, the following indirect method of calculating $n_{2}$ was employed.

Applying the perfect-gas equation to the open system raised to a higher temperature, $T_{2}$, the following relation is obtained:

where

$$
P_{1} V_{1}=n_{2} R T_{2},
$$

$P_{1}=$ the atmospheric pressure.

$V_{1}=$ the volume of the system.

$n_{2}=$ the number of moles of air left in the system at system temperature $T_{2}$.

If the system is closed at room temperature and pressure, and the platinum coil then heated sufficiently to raise the system temperature to $T_{2}$, the situation is described by

where

$$
P_{2} V_{1}=n_{1} R T_{2},
$$

$P_{2}=$ the pressure at system temperature $T_{2}$.

$V_{1}=$ the volume of the system.

$n_{1}=$ the original number of moles of air in the system (room temperature and atmospheric pressure).

Dividing eq (3) by eq (4),

$$
\frac{P_{1}}{P_{2}}=\frac{n_{2}}{n_{1}},
$$

and $n_{2}$ may be calculated from an experimental measurement of the pressure developed when the closed system is heated to temperature $T_{2}$.

For the purposes of this investigation it was unnecessary to know the actual value of the system temperature at which the determinations were made. Hence, it was sufficient to measure the pressure developed in the closed system when a selected heating-coil temperature had been reached, and to calculate the value of $n_{2}$ produced by those conditions without further defining them. In turn, $X_{2}$ can be calculated from $n_{2}$ by means of eq (1). To avoid a separate measurement of this pressure at the beginning of each run, a calibration curve of pressure versus heating-coil temperature was made, and the closed-system pressure for the run temperature selected was read from the curve. In practice, therefore, to avoid pressure buildup before combustion, the drawoff tube could be left open during the initial period of heating the equipment.

This method of avoiding a preliminary pressure buildup was considered preferable to one that had been used by other workers, which utilized a balloon into which the gases expanded [1]. A balloon introduces errors arising from (a) the difficulty of washing adsorbed gases, as hydrogen chloride, from the fabric; (b) the possible introduction of organic vapors into the samples; and (c) the diffusion of gases through the fabric.

At the beginning of each run the specimen was placed in a porcelain combustion boat, which was placed in the silica tube as far from the platinum coil as possible. Then the 2-liter glass bulb was placed in position and the two springs attached. The combustion chamber was cooled by the air coming from the jets shown in figure 1 .

The combustion chamber was evacuated by opening the stopcock to the vacuum manifold. This evacuation removed any organic vapors that might have been present from a preceding run. After evacuation, the vacuum stopcock was closed and the drawoff valve opened to the atmosphere. Current was then applied to the platinum coil.

When the initial combustion temperature, as indicated by the thermocouple, was attained, the drawoff tube was closed. Then the combustion chamber was inclined, causing the combustion boat containing the specimen to slide down the silica tube into the hot platinum coil, which ignited the specimen.

The input to the heating coil was maintained constant for $12 \mathrm{~min}$, the current was turned off, and the apparatus was allowed to cool to room temperature. The gases were then collected as described below.

Several sample bulbs having volumes of $50 \mathrm{ml}$ and $2,000 \mathrm{ml}$ were constructed by sealing vacuum stopcocks and 19/38 ground-glass female joints to bulbs of appropriate size. The $50-\mathrm{ml}$ bulbs were used for the mass-spectrometer analyses, and the $2,000-\mathrm{ml}$ bulbs were used for the carbon monoxide determinations. Before a run, the sample bulb used was evacuated by attaching it to the vacuum manifold. The apparatus was cooled to room temperature, and then the evacuated sample bulb was placed on the drawoff arm and the drawoff stopcock opened. Approximately one-fourth of the air was immediately transferred out of the drawoff arm by expansion of the air from $1 \mathrm{~atm}$ to roughly $1 / 2 \mathrm{~atm}$. Assuming negligible subsequent mixing, the above procedure would lead to dilution of the combustion gases with 
about 8 percent of air. In order to allow subsequent diffusive mixing, the sample stopcock was not opened until 10 min after the drawoff arm was opened. Thus, the actual contamination, although unknown, was probably somewhat less than 8 percent. For the colorimetric carbon monoxide determinations the error introduced by this method was negligible. Both stopcocks were then closed, and the sample bulb was attached either to the mass spectrometer or to the carbon monoxide apparatus.

After the combustion chamber cooled to room temperature, a partial vacuum was invariably present within the apparatus. In those cases where hydrogen chloride was expected, the drawoff arm was attached, by means of a piece of plastic tubing, to a beaker containing dilute $(p \mathrm{H}=10)$ sodium hydroxide. This solution was drawn into the combustion chamber and allowed to stand for $15 \mathrm{~min}$. Due to the high affinity of hydrogen chloride gas for water, any of this gas not adsorbed upon the vessel walls was expected to dissolve in the base. After $15 \mathrm{~min}$ the springs were removed, and the solution was poured into a beaker. The combustion chamber was then washed several times with distilled water, and these water washings were combined and analyzed by the method described below.

\section{Analyses}

The 50-ml sample bulb containing combustion gases was placed on the inlet system of the mass spectrometer. An analysis was carried out for gases having a mass number of 1 to 100 . The analytical results are recorded in table 1 .

Carbon monoxide was determined by two methods. When it was present in concentrations above 1,000 $\mathrm{ppm}$, the mass spectrometer was used. When present in concentrations below $1,000 \mathrm{ppm}$, the mass spectrometer was not sensitive enough to give an accurate analysis. It was, therefore, necessary to use the indicator technique [6]. The 2,000-ml sample bulb was filled with combustion gases, and the pressure reading on the manometer was recorded. The bulb was attached to a source of carbon monoxide-free air under pressure, and this air was allowed to enter the bulb until the pressure was slightly above $1 \mathrm{~atm}$. Then the bulb was attached to an indicator tube [6], and the gases passed through the indicator at a constant rate, as determined by a flowmeter. At the end of a given time period, the color of the indicator was compared with a color chart, and the concentration of carbon monoxide in the sample bulb was determined.

TABLE 1. Results obtained upon burning combustible materials in air

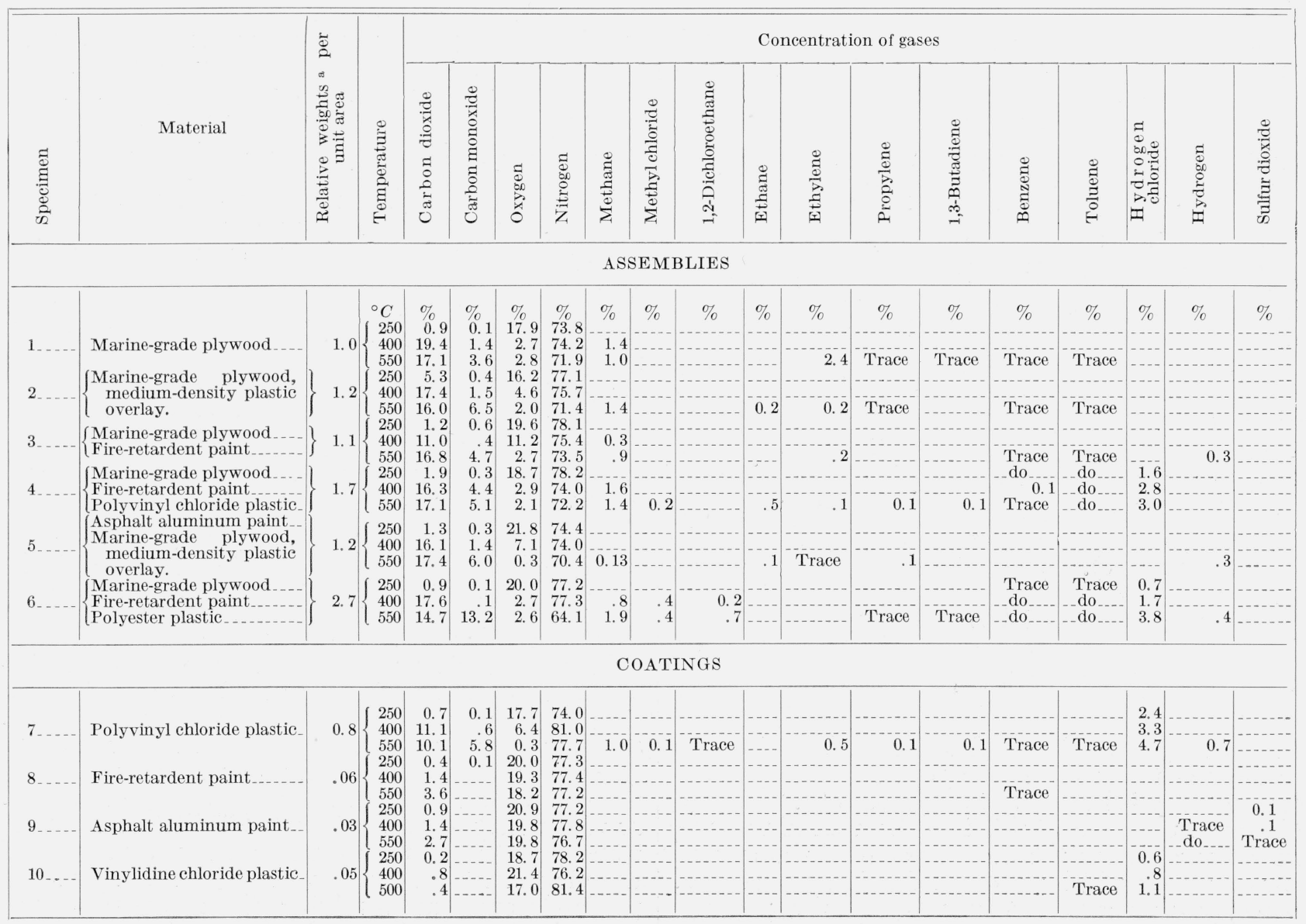

a This column is the ratio of the weight of each material for a certain area to the weight of the exterior plywood for the same area. 
Hydrogen chloride was adsorbed upon the walls of the combustion chamber, along with water produced during the course of combustion. Because of this adsorption, it was not possible to determine hydrogen chloride by means of the mass spectrometer. Instead, the water washings used in the procedure outlined previously were adjusted to a $p \mathrm{H}$ of 6.0 to 7.0 and titrated with standard $0.1 \mathrm{~N}$ silver nitrate, using fluorescein as an indicator [7].

\section{Materials}

To illustrate the utility of this method, several samples of organic wall and ceiling coatings were burned, and an analysis of their gaseous combustion products was carried out. These materials are listed in table 1.

In order to duplicate field conditions, the volume of a structure in field use was calculated, as was the area of the walls and ceiling of this structure. The area of each specimen was then adjusted so that the ratio of the specimen area to the volume of the combustion chamber was equal to the ratio of the ceiling and wall area to the volume of the structure.

\section{Results and Discussion}

The results of the analyses of the combustion gases are recorded in table 1.

Each material was organic, and gave rise to carbon monoxide and carbon dioxide upon combustion. The plywood apparently contained only carbon, hydrogen, and oxygen, and only gases composed of these elements were detected.

The plastic coatings contained chlorine as well as carbon, hydrogen, and oxygen, and gave rise to hydrogen chloride and chlorinated hydrocarbons, in addition to carbon monoxide and carbon dioxide. Hydrogen chloride has been found in the combustion gases of several types of chlorinated plastics, although there appears to be little information on the presence of chlorinated hydrocarbons [1].

The sulfur dioxide from the combustion of the asphalt aluminum paint probably came from the asphalt-resin base. It is known that the sulfur content of asphalt can be as high as 5 percent, depending upon its source [8]. No sulfur dioxide was found when the plywood material surfaced with the asphalt aluminum paint was burned (specimen 5). This was probably due to the adsorption of the sulfur dioxide, by the water formed upon combustion of the plywood, on the walls of the combustion chamber.

For every material the greatest variety of gases occurred at the highest initial temperature $\left(550^{\circ} \mathrm{C}\right.$.). At this temperature, cracking and decomposition occurred, and small molecular fragments, such as methane and hydrogen, were formed from many of the specimens.

The chlorinated plastics liberated chlorinated hydrocarbons at the higher temperatures, whereas the only chlorinated material produced at the lowest temperature was hydrogen chloride. In each case the amount of hydrogen chloride increased as the temperature increased.

The assemblies and the polyvinyl chloride plastic coating are the heaviest materials listed in table 1. The quantities used were sufficient to consume all the oxygen if they were to burn entirely to carbon dioxide and water. As might be expected, these materials produced the largest concentrations of combustion gases, and caused the greatest decrease in the oxygen concentration.

For these materials, the combustion gases had oxygen concentrations of 0.3 to 3 percent at the highest initial temperature. This low oxygen concentration is typical not only of the closed system used here, but also of large, open, ventilated systems.

For example, Kingman [5] and Olsen [2] report very low oxygen concentrations as the result of analyses of the combustion gases of ventilated and unventilated burning rooms. In general, the gases produced in most fires are the products of combustion and destructive distillation on decomposition [2]. In the method described in this paper, the above condition was approximated by forced heating of the specimen, after the oxygen concentration had dropped to a level below which flames could be sustained.

The foregoing procedure, while not capable of extreme accuracy, will give a quantitative estimate of the concentrations of combustion gases produced from commercially available organic coatings. Such information is of importance when designing a dwelling for use under certain conditions, and in producing organic coatings with special thermal-breakdown properties.

The author thanks the following Bureau staff members: S. Gunther, F. Elder, and S. Schuhmann for the mass-spectrometer analyses and the carbon monoxide determinations, and J. Loftus for the hydrogen chloride determinations.

\section{References}

[1] E. H. Coleman and C. H. Thomas, J. Appl. Chem. 4, 379 (1954).

[2] J. C. Olsen, G. E. Ferguson, and L. Scheflan, Ind. Eng. Chem. 25, 599 (1933)

[3] L. B. Berger, H. A. Schrenk, I. A. Gale, R. W. Stewart, and L. E. Sieffert, U. S. Bureau of Mines Rept. R. I. 4134 (Oct. 1947).

[4] Fire Eng. 99, 148 (1946).

[5] F. E. T. Kingman, E. H. Coleman, and D. S. Rasbash, J. Appl. Chem. 3, 463 (1953).

[6] M. Shepherd, Anal. Chem. 19, 77 (1947).

[7] I. M. Kolthoff and E. B. Sandell, Textbook of quantitative analyses, p. 475, 572 (The Macmillan Co., New York, N. Y., 1949).

[8] H. Abraham, Asphalts and allied substances, p. 73 (D. Van Nostrand Co., New York, N. Y., 1945).

Washington, May 17, 1956. 